This item was submitted to Loughborough's Research Repository by the author.

Items in Figshare are protected by copyright, with all rights reserved, unless otherwise indicated.

\title{
Optical properties of graphene-based materials in transparent polymer matrices
}

\section{PLEASE CITE THE PUBLISHED VERSION}

http://dx.doi.org/10.1063/1.4961674

\section{PUBLISHER}

(C) The Authors. Published by American Institute of Physics (AIP)

\section{VERSION}

NA (Not Applicable or Unknown)

\section{PUBLISHER STATEMENT}

This work is made available according to the conditions of the Creative Commons Attribution-NonCommercialNoDerivatives 4.0 International (CC BY-NC-ND 4.0) licence. Full details of this licence are available at: https://creativecommons.org/licenses/by-nc-nd/4.0/

\section{LICENCE}

CC BY-NC-ND 4.0

\section{REPOSITORY RECORD}

Bayrak, Osman, Mariana Ionita, Emrah Demirci, and Vadim Silberschmidt. 2019. "Optical Properties of Graphene-based Materials in Transparent Polymer Matrices”. figshare. https://hdl.handle.net/2134/22434. 


\section{Optical properties of graphene-based materials in transparent polymer matrices}

Osman Bayrak, Mariana Ionita, Emrah Demirci, and Vadim V. Silberschmidt

Citation: Appl. Phys. Lett. 109, 081905 (2016); doi: 10.1063/1.4961674

View online: $\mathrm{http}: / / \mathrm{dx}$.doi.org/10.1063/1.4961674

View Table of Contents: http://aip.scitation.org/toc/apl/109/8

Published by the American Institute of Physics

\section{Articles you may be interested in}

$\mathrm{ZnO}$ nanolasers on graphene films

Applied Physics Letters 108, 263102 (2016); 10.1063/1.4954798

PEDOT:PSS with embedded $\mathrm{TiO}_{2}$ nanoparticles as light trapping electrode for organic photovoltaics Applied Physics Letters 108, 253302 (2016); 10.1063/1.4954902

Patterning monolayer graphene with zigzag edges on hexagonal boron nitride by anisotropic etching Applied Physics Letters 109, 053101 (2016); 10.1063/1.4959963

All-dielectric phase-change reconfigurable metasurface Applied Physics Letters 109, 051103 (2016); 10.1063/1.4959272

Highly photoresponsive charge-sensitive infrared phototransistors with a dynamically controlled optical gate Applied Physics Letters 109, 091102 (2016); 10.1063/1.4961938

Efficient and versatile graphene-based multilayers for EM field absorption Applied Physics Letters 109, 093103 (2016); 10.1063/1.4962148

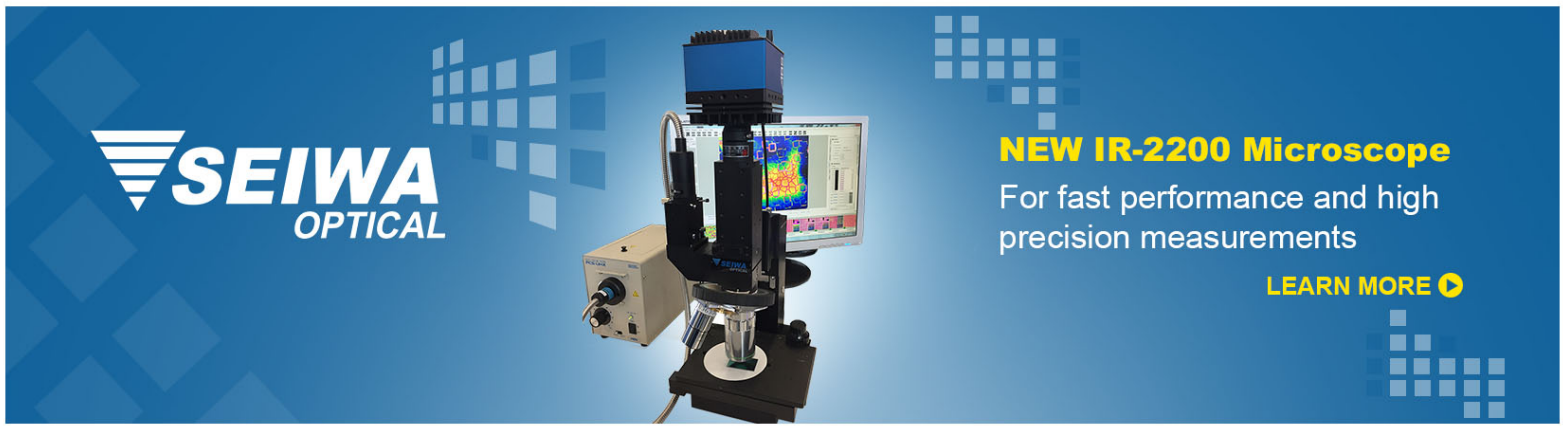




\title{
Optical properties of graphene-based materials in transparent polymer matrices
}

\author{
Osman Bayrak, ${ }^{1}$ Mariana Ionita, ${ }^{2}$ Emrah Demirci, ${ }^{1, a)}$ and Vadim V. Silberschmidt ${ }^{1}$ \\ ${ }^{1}$ Wolfson School of Mechanical, Electrical and Manufacturing Engineering, Loughborough University, \\ Loughborough, LE11 3TU, United Kingdom \\ ${ }^{2}$ Advanced Polymer Materials Group, University Politehnica of Bucharest, 132 Calea Grivitei, \\ 010737 Bucharest, Romania
}

(Received 2 June 2016; accepted 15 August 2016; published online 26 August 2016)

\begin{abstract}
Different aspects of graphene-based materials (GBMs) and GBM-nanocomposites have been investigated due to their intriguing features; one of these features is their transparency. Transparency of GBMs has been of an interest to scientists and engineers mainly with regard to electronic devices. In this study, optical transmittance of structural, purpose-made nanocomposites reinforced with GBMs was analyzed to lay a foundation for optical microstructural characterization of nanocomposites in future studies. Two main types of GBM reinforcements were studied, graphene oxide (GO) and graphite nanoplates (GNPs). The nanocomposites investigated are GO/poly(vinyl alcohol), GO/sodium alginate, and GNP/epoxy with different volume fractions of GBMs. Together with UVvisible spectrophotometry, image-processing-assisted micro and macro photography were used to assess the transparency of GBMs embedded in the matrices. The micro and macro photography methods developed were proven to be an alternative way of measuring light transmittance of semitransparent materials. It was found that there existed a linear relationship between light absorbance and a volume fraction of GBMs embedded in the same type of polymer matrices, provided that the nanocomposites of interest had the same thicknesses. This suggests that the GBM dispersion characteristics in the same type of polymer are similar and any possible change in crystal structure of polymer due to different volumetric contents of GBM does not have an effect on light transmittance of the matrices. The study also showed that the same types of GBMs could display different optical properties in different matrix materials. The results of this study will help to develop practical microstructural characterization techniques for GBM-based nanocomposites. Published by AIP Publishing. [http://dx.doi.org/10.1063/1.4961674]
\end{abstract}

Among its many outstanding physical properties, optical transparency of graphene is important for applications in electronic devices and transparent coatings. ${ }^{1}$ Graphene and its derivatives, shortly graphene-based materials (GBMs), can provide strength, transparency, and impermeability at the same time when used as a reinforcement material for nanocomposites. ${ }^{2}$ Having tunable optical properties, GBMs were shown to have a potential to be used in optoelectronic applications. $^{3,4}$

Optical contrast of multi-layer graphene (MLG) alters with change in the number of layers, making it possible to determine a number of layers in a given sample. ${ }^{5}$ According to Nair et al., ${ }^{6}$ a single-layer pristine graphene transmits $97.7 \%$ of white light and only $0.1 \%$ of it is reflected. Zhu et al. derived a formula that provides a number of layers in a multi-layer graphene (MLG) based on its light transmittance and showed that white-light transmission through MLG depended only on the number of layers within the flake. ${ }^{7}$ Kasry et al. indicated that a link between a layer number and transmission for graphene could obviously obey the BeerLambert law. ${ }^{8}$ Due to inherent structural defects, graphene oxide (GO) can transmit more light than pristine graphene does. A study showed that $9 \mathrm{~nm}$-thick GO sheets transmit $96 \%$ of light of $550 \mathrm{~nm}$ wavelength. ${ }^{9}$ The same study reported that the light transmittance decreased after partial

${ }^{a)}$ E.Demirci@lboro.ac.uk restoration of the structural defects with reductive treatments that produced reduced $\mathrm{GO}(\mathrm{rGO})$.

Kim et al. manufactured transparent and gas-barrier GO/ poly(vinyl alcohol) (PVA) nanocomposite films. ${ }^{10}$ Transparent and electrically conductive $\mathrm{rGO} /$ silica nanocomposites were reported by Watcharotone et al. ${ }^{11}$ Manufacturing methods of the nanocomposites, used in both studies, were based on solution mixing that led to the formation of flakes aligned parallel to the plane of the films. This allowed the light beams to pass through the flakes almost orthogonally. Both studies reported that nanoparticles were uniformly distributed in the matrices and transparency of the nanocomposites reduced with increased GO content. Gan et al. showed that the same types of GBM nanoparticles with different geometries may induce different features of optical transmittance in polymers they are mixed with. ${ }^{12}$

In this paper, optical properties of GBM sheets inside different matrix materials are researched. This study was planned as a preliminary analysis to enable an optical microstructural characterization technique for GBM-based nanocomposites. GO/poly(vinyl alcohol) (GO/PVA), GO/sodium alginate (GO/SA), and GNP/epoxy nanocomposites with different volume fractions of nanofillers were used. All of the studied nanocomposites were manufactured with a type of solution-mixing method resulting in samples with GBM layers preferentially aligned parallel to the plane of the nanocomposites. A UV-visible spectrophotometer was used for 
GO/PVA and GNP/Epoxy nanocomposites. Optical microscopy (micro photography) and macro photography were also employed to assess their potential for measuring the optical properties of nanocomposites. It was found that optical transmittance at the green wavelength of $550 \mathrm{~nm}$, obtained with the photography methods used for GO/PVA and GNP/epoxy nanocomposites, matched the values obtained using the spectrophotometer with an acceptable accuracy. A relationship between the volume content and light absorbance of the GBMs was demonstrated. Absorbance per single GBM layer in different matrices is also discussed.

Three different types of GBMs were analyzed in this study. A film of pure SA and GO/SA nanocomposite films with GO volume fractions of $0.175 \%, 0.466 \%$, and $1.167 \%$ were manufactured as reported by Ionita et al. ${ }^{13}$ Pure epoxy and GNP/epoxy nanocomposites with GNP volume fractions of $0.056 \%$ and $0.287 \%$ were kindly supplied by Professor Colak and Mr. Acar (Yildiz Technical University, Turkey); their manufacturing procedure is discussed in Ref. 14. GO/ PVA nanocomposite films with GO volume fractions of $0.066 \%, 0.198 \%, 0.464 \%, 0.663 \%$, and $1.33 \%$ were manufactured as following. GO was purchased from the National Institute for Research and Development in Microtechnologies (Romania) and prepared following the Hummers method. PVA (130000 g/mol MW, +99\% hydrolyzed) was supplied by Sigma Aldrich. 1 wt. \% PVA pellets were added in portions to distilled water under constant stirring. Then, the polymer was dissolved by means of autoclaving at $120^{\circ} \mathrm{C}$ for $60 \mathrm{~min}$. A volume of $50 \mathrm{ml}$ of the obtained $1 \mathrm{wt}$. \% PVA solution was mixed with GO with various weights $(0.0005,0.0015,0.0035$, 0.005 , and $0.01 \mathrm{~g}$ ). The mixtures were subjected to ultrasound treatment for $60 \mathrm{~min}$ in an ice bath. This was performed using a VCX750 ultrasonic processor (Sonics \& Materials, Inc.) equipped with a titanium-alloy (Ti-6Al-4V) probe tip and a $750 \mathrm{~W}$ power source operating at a frequency of $20 \mathrm{kHz}$. The homogenized GO/PVA nanocomposite solutions were poured into transparent Petri dishes and let drying for $72 \mathrm{~h}$ at room temperature. The PVA film and GO/PVA nanocomposite films were manufactured in $100 \mu \mathrm{m}$ thickness. The pure SA film and GO/SA nanocomposite films had a thickness of $50 \mu \mathrm{m}$. Pure epoxy and GNP/epoxy nanocomposites were supplied in $\sim 2.5 \mathrm{~mm}$ thickness. To allow visible light to pass through the GNP/epoxy samples, they were thinned down to $720 \mu \mathrm{m}$ using a milling machine.

The measurements of optical transmittance were first done using a UV-visible spectrophotometer, Hewlett Packard 8453. The pure PVA and epoxy films without any GBM inclusions were used as blank samples. The reason for using the pure matrix materials was to extract the optical properties of GBMs inside these matrices, rather than those of the nanocomposites. Optical microscopy with narrowband filters was shown before to be suitable for obtaining optical transmittance of graphene. ${ }^{6}$ In addition to the spectrophotometer, back-illuminated optical microscopy with a green-light filter was used in this study for the nanocomposites, which is a similar method used in Ref. 6 for graphene. The optical microscope used for this study was LEICA DM6000 M with a built-in digital camera. Settings of the microscope camera were kept the same for the same type of nanocomposites. Also, back-illuminated macro photography with the same green-light filter was used in this study. Micro and macro photographs of the nanocomposites were analyzed with an in-house image-processing software. In the macro photography, back-illumination was provided by a high-definition smartphone screen with a white-color object (grey-scale value of 255). The green light filter (COMAR 550 IL50 T-FOY) with a wavelength band of $550 \pm 5 \mathrm{~nm}$ was placed on top of the screen, and the samples were positioned on top of the filter. A cardboard box that can house the setup was employed to prevent any other light coming onto the samples. A small opening, through which a digital camera could be inserted, was made on top of the box. Shining parts of the camera that might cause reflection of the screen light were covered with black, non-reflective layers. The camera was placed on the hole as to see the sample underneath. Photographs were taken with manual ISO, diaphragm, aperture, and exposure time settings of the camera. The same settings of the camera and the same level of brightness of the illumination screen were maintained for the same class of nanocomposites. The settings were chosen in a way that all the samples of the same type of composites could be recognized, i.e., neither invisible because of the strong light nor completely dark due to weak light. The ISO value of the camera was kept at the minimum to get images with least noise. The macro photographs taken are presented in Fig. 1(a). An in-house image-processing software was used to extract average green pixel values from the micro and macro photographs. As in the spectrophotometer, the pure polymer films were used as blank samples. The following formula was used to obtain optical transmittance properties of the samples from the photos

$$
\% T_{G r}=\frac{P V_{\text {composite }} \times 100}{P V_{\text {polymer }}},
$$

where $P V$ and $T_{G r}$ stand for the average green pixel value and transmittance, respectively. Optical transmittance of the GO/PVA nanocomposite films was measured using the spectrophotometer, micro photography, and macro photography. In the used procedure. the depth of the field of the used microscope lens covered the whole thickness of the films.

The transmittance values obtained using the spectrophotometer in the visible-light range and those with the wavelength of $550 \mathrm{~nm}$ obtained with micro and macro photography are plotted in Fig. 1(b). More than one measurement was done on the samples. Discrepancy between the results was found to be less than $10 \%$ for the spectrophotometry measurements and $1 \%$ for the photography measurements. As can be seen in the figure, the values obtained with the micro and macro photography matched the results acquired with the spectrophotometer. A similar analysis was carried out for the GNP/epoxy nanocomposites. However, micro photography could not be implemented for the GNP/ epoxy samples, since they were much thicker than the depth of the field of the used microscope lenses. Transmittance of the GNP nanoflakes inside GNP/epoxy nanocomposites is plotted in Fig. 1(c). (Due to an error caused by the spectrophotometer in a wavelength range of $640 \mathrm{~nm}$ to $670 \mathrm{~nm}$, the curves were not plotted beyond $640 \mathrm{~nm}$ as the error resulted in large fluctuations between $640 \mathrm{~nm}$ and $670 \mathrm{~nm}$.) As in the 

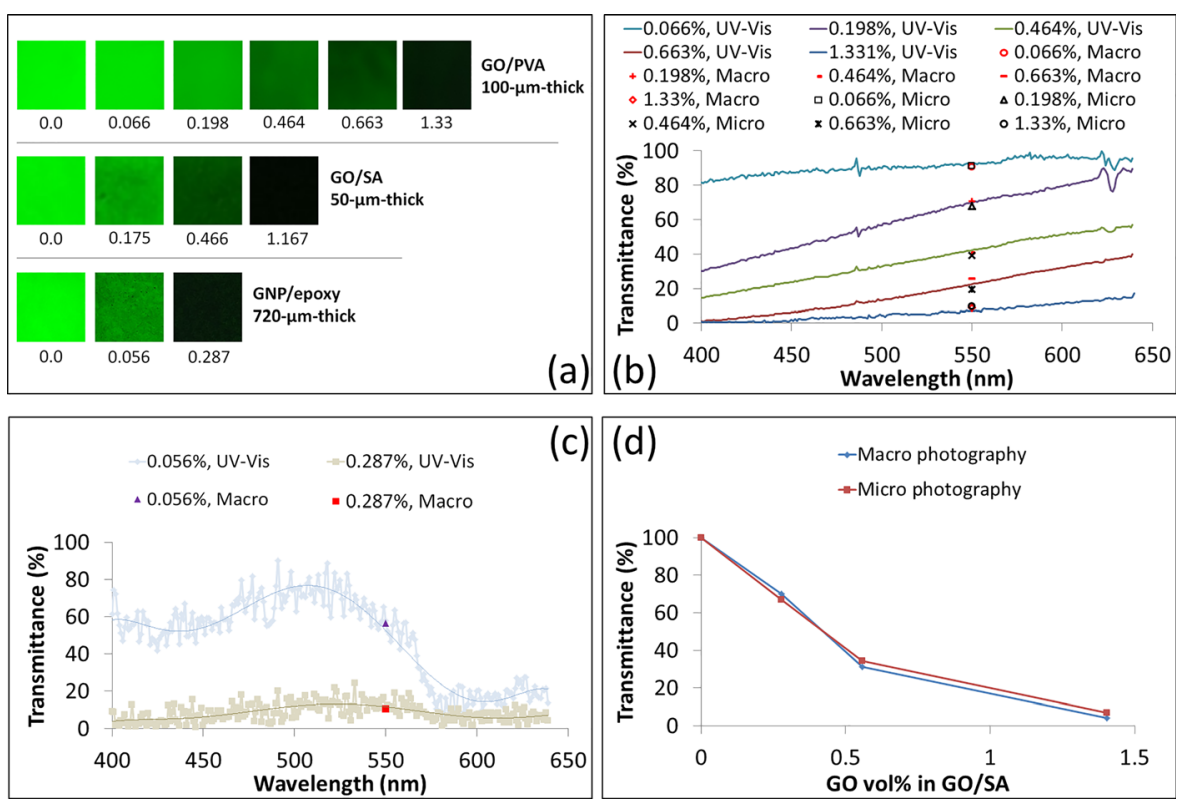

FIG. 1. (a) Macro photographs of samples, depicted with volume fractions of GBM (images are around $5 \mathrm{~mm} \times$ $5 \mathrm{~mm}$ ). Optical transmittance of GBM particles: (b) in GO/PVA nanocomposites, obtained with UV-visible spectrophotometer (continuous curves), macro photography (red markers), and micro photography (black markers); (c) in GNP/epoxy nanocomposites, obtained with UV-visible spectrophotometer (continuous curves) and macro photography (markers); (d) in GO/SA nanocomposites, obtained with micro and macro photography. case of GO/PVA, the alternative methods of the transmittance measurement worked well for GNP/epoxy. Both figures show that optical transmittance of GBM particles decreased as their content increased in polymer matrices. Apparently, the micro and macro photography techniques implemented gave acceptable transmittance values at certain ranges for the studied nanocomposites.

Optical transmittance of GO flakes inside the SA matrix could not be obtained using the UV-visible spectra since only small volumes of nanocomposites were available. The aperture for the light beams of the spectrophotometer was larger than the samples; this led to erroneous results. Therefore, these nanocomposites were tested with the micro and macro photography techniques only. The optical transmittance values at the wavelength of $550 \mathrm{~nm}$ are plotted in Fig. 1(d). As can be seen from the obtained results, the transmittance values obtained with these methods match with a reasonable accuracy.

A relationship between light absorbance and volume fraction of GBM flakes in the polymer matrices at the wavelength of $550 \mathrm{~nm}$ was also researched. The transmittance values were converted into absorbance magnitudes using a $2-\log \left(\% T_{G r}\right)$ link. Since the transmittance values obtained with different methods were close to each other, the absorbance curves were plotted based on the data from one method. For GO/SA and GO/PVA, the data from the micro photography were used. The macro photography was employed for GNP/epoxy as the micro photography was not suitable for it. As the thickness magnitudes for three different classes of nanocomposites were not the same, the obtained results were not plotted in the same absorbanceGBM volume fraction graph. As can be seen in Figs. 2(a),
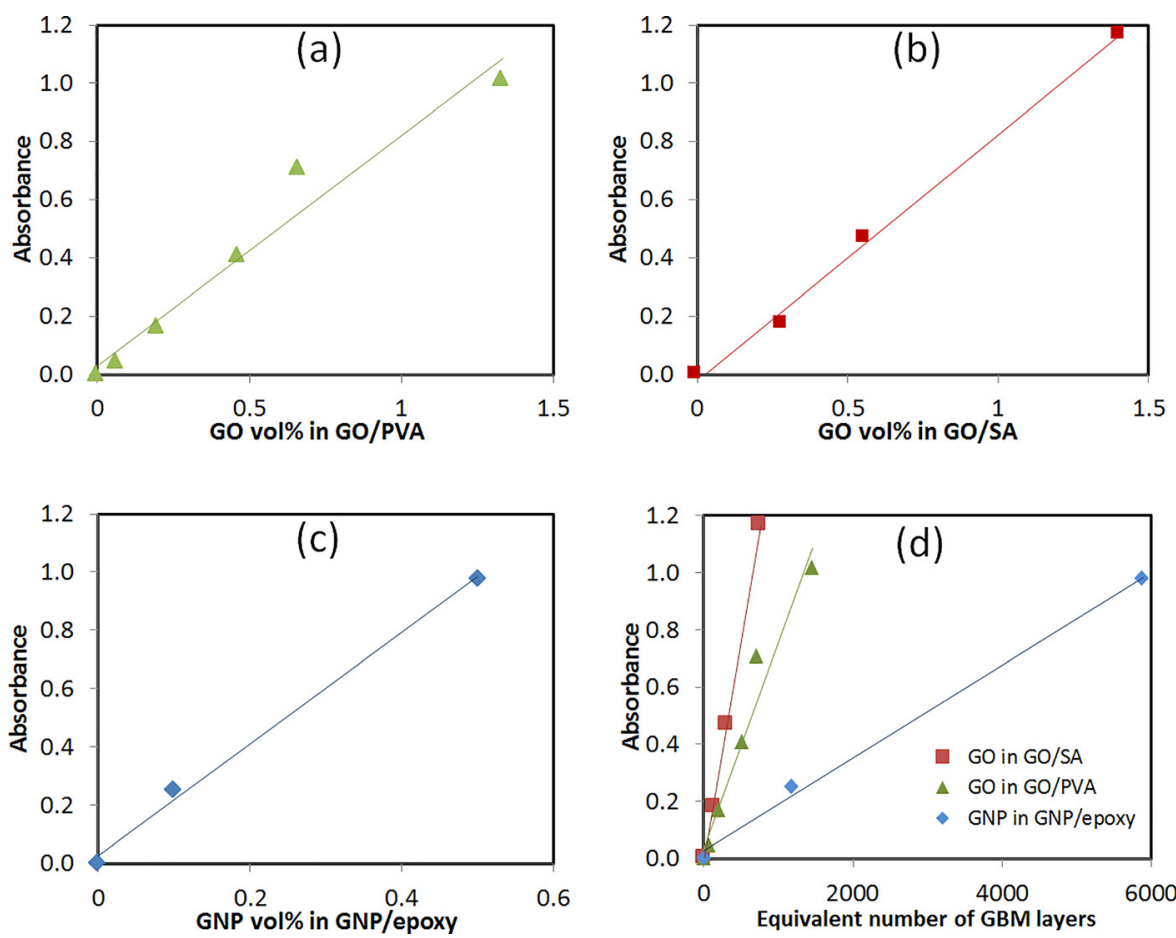

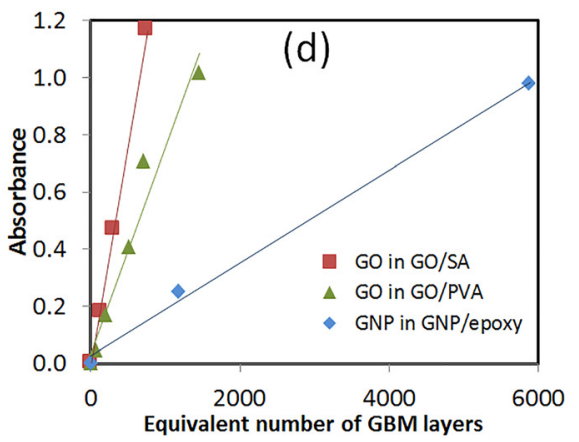

FIG. 2. Light absorbance by GBM particles as a function of their volume fraction in PVA (a), SA (b), and epoxy (c) matrices. (d) Absorbance values as a function of equivalent numbers of GBM layers through the thickness of nanocomposites. 
2(b), and 2(c), there is an almost-linear relationship between the volume fraction of the GBM flakes in the used polymers and their absorbance values. This character of relationship means that dispersion of the GBMs inside the polymer matrix obeyed the Beer-Lambert law, implying a similar dispersion characteristic of GBM within the same class of nanocomposites. Importantly, to conclude that any possible change in the microstructure of the polymer with different GBM contents would have no effect on light transmittance of the nanocomposites.

It is known that the solution mixing methods that are also employed to manufacture the nanocomposites used in this study lead to dispersion of nanoflakes mostly parallel to the moulding plane. Also, the absorbance-volume fraction relations showed that the absorbance increased linearly with the increase in the GBM volume content. From these, it can be deduced that the number of GBMs in the pathway of the light, i.e., through the thickness of the nanocomposites, changed linearly with the change of the volume fraction (This may not apply to much thinner films; the minimum sample thickness used in this study was $50 \mu \mathrm{m}$ ). As a result, curves for absorbance as a function of a number of GBM layers in different matrices could be plotted. A notion "equivalent number of GBM layers" was introduced to plot all the curves in a single graph as shown in Fig. 2(d). When calculating the equivalent number of GBM layers $(E N L)$, the layers were assumed to stretch through the entire width of the nanocomposites and parallel to their plane. To calculate the $E N L$, the following equation was used:

$$
E N L=\frac{v f \times t_{n c}}{t_{G B M}},
$$

where $v f$ is the volume fraction of GBMs in each nanocomposite, $t_{n c}$ is the thickness of the nanocomposites, and $t_{G B M}$ is the thickness of the GBM layer. The calculated results in Fig. 2(d) demonstrate that the same type of GBMs used in different matrices might have a different absorbance feature. Several reasons can be suggested to explain this result. First, dispersion characteristics, that may be different in different matrices, can affect the absorption. Second, a chemical structure of GO changes with the covalent bonds established with matrices; it means that different matrices might have caused different chemical structures with GO. Third, Van der Waals interaction between graphene and the matrix can alter the optical properties of graphene. Stauber et al. showed a significance of interlayer interaction for optical properties of graphene. ${ }^{15}$ Also, it is known that the electron structure of graphene affects its light-absorbance property. Van-derWaals interaction between graphene-like substances and polymer has an effect on the electron structure. ${ }^{16,17}$ Therefore, a change in the electron structure of nanoflakes caused by such interactions with the matrix is assumed to be another reason. Fourth, as reported by $\mathrm{Ni}$ et al. ${ }^{18}$ a strain of graphene may change as a result of interaction with a substance in contact; this strain change also affects optical properties of graphene. Therefore, it can be suggested that any residual strain on the GBMs potentially induced by the manufacture of the nanocomposites could change the absorbance properties of the GBM flakes inside the matrix.

In summary, this study demonstrated that micro and macro photography can be used as a suitable way to evaluate optical properties of GBM-nanocomposites. A linear correlation between light absorbance and a volume fraction of GBM particles in transparent matrix media was found. This implies that dispersion characteristics of polymers with different concentrations of GBMs in the same matrix are similar. Any possible change in a microstructure of the polymer related to different concentrations of GBMs does not affect the light transmittance of the nanocomposites. It was also demonstrated that the same type of GBM might have different optical properties in different matrices. The results obtained in this study will help to develop more practical microstructural characterization techniques for GBM-based nanocomposites.

The authors would like to thank Professor Ozgen Umit Colak and Alperen Acar (Yildiz Technical University, Istanbul/ Turkey) for kindly providing GNP/epoxy nanocomposites.

${ }^{1}$ K. S. Novoselov, V. I. Fal'ko, L. Colombo, P. R. Gellert, M. G. Schwab, and K. Kim, Nature 490, 192 (2012).

${ }^{2}$ I. H. Tseng, Y. F. Liao, J. C. Chiang, and M. H. Tsai, Mater. Chem. Phys. 136, 247 (2012).

${ }^{3}$ W. Zhu, I. D. Rukhlenko, and M. Premaratne, Appl. Phys. Lett. 102, 241914 (2013).

${ }^{4}$ R. Maiti, A. Midya, C. Narayana, and S. K. Ray, Nanotechnology 25, 495704 (2014).

${ }^{5}$ P. Blake, E. W. Hill, A. H. Castro Neto, K. S. Novoselov, D. Jiang, R. Yang, T. J. Booth, and A. K. Geim, Appl. Phys. Lett. 91, 063124 (2007).

${ }^{6}$ R. R. Nair, P. Blake, A. N. Grigorenko, K. S. Novoselov, T. J. Booth, T. Stauber, N. M. R. Peres, and A. K. Geim, Science 320, 1308 (2008).

${ }^{7}$ S. E. Zhu, S. J. Yuan, and G. C. A. M. Janssen, Europhys. Lett. 108, 17007 (2014).

${ }^{8}$ A. Kasry, M. A. Kuroda, G. J. Martyna, G. S. Tulevski, and A. A. Bol, ACS Nano 4, 3839 (2010).

${ }^{9}$ H. A. Becerril, J. Mao, Z. Liu, R. M. Stoltenberg, Z. Bao, and Y. Chen, ACS Nano 2, 463 (2008).

${ }^{10}$ H. M. Kim, J. K. Lee, and H. S. Lee, Thin Solid Films 519, 7766 (2011).

${ }^{11}$ S. Watcharotone, D. A. Dikin, S. Stankovich, R. Piner, I. Jung, G. H. B. Dommett, G. Evmenenko, S. E. Wu, S. F. Chen, C. P. Liu, S. T. Nguyen, and R. S. Ruoff, Nano Lett. 7, 1888 (2007).

${ }^{12}$ Y. Gan, M. Feng, and H. B. Zhan, Appl. Phys. Lett. 104, 171105 (2014).

${ }^{13}$ M. Ionita, M. A. Pandele, and H. Iovu, Carbohydr. Polym. 94, 339 (2013).

${ }^{14}$ A. Acar, O. U. Colak, and D. Uzunsoy, Mater. Test. 57, 1001 (2015).

${ }^{15}$ T. Stauber, N. M. R. Peres, and A. K. Geim, Phys. Rev. B 78, 085432 (2008).

${ }^{16}$ E. R. Badamshina and M. P. Gafurova, Polym. Sci., Ser. B 50, 215 (2008).

${ }^{17}$ L. V. Vinogradova, E. Y. Melenevskaya, A. S. Khachaturov, E. E. Kever, L. S. Litvinova, A. V. Novokreshchenova, M. A. Sushko, S. I. Klenin, and V. N. Zgonnik, Vysokomol. Soedin. 40, 1854 (1998), http://cat.inist.fr/ ?aModele $=$ afficheN\&cpsidt $=9993464$.

${ }^{18}$ Z. H. Ni, W. Chen, X. F. Fan, J. L. Kuo, T. Yu, A. T. S. Wee, and Z. X. Shen, Phys. Rev. B 77, 115416 (2008). 